\title{
Evaluation Framework for Real-Time Adaptive 360-Degree Video Streaming over 5G Networks
}

\author{
Cise Midoglu \\ Simula Research Laboratory \\ cise@simula.no
}

\author{
Òzgü Alay \\ Simula Metropolitan Center for \\ Digital Engineering \\ ozgu@simula.no
}

\author{
Carsten Griwodz \\ University of Oslo \\ griff@ifi.uio.no
}

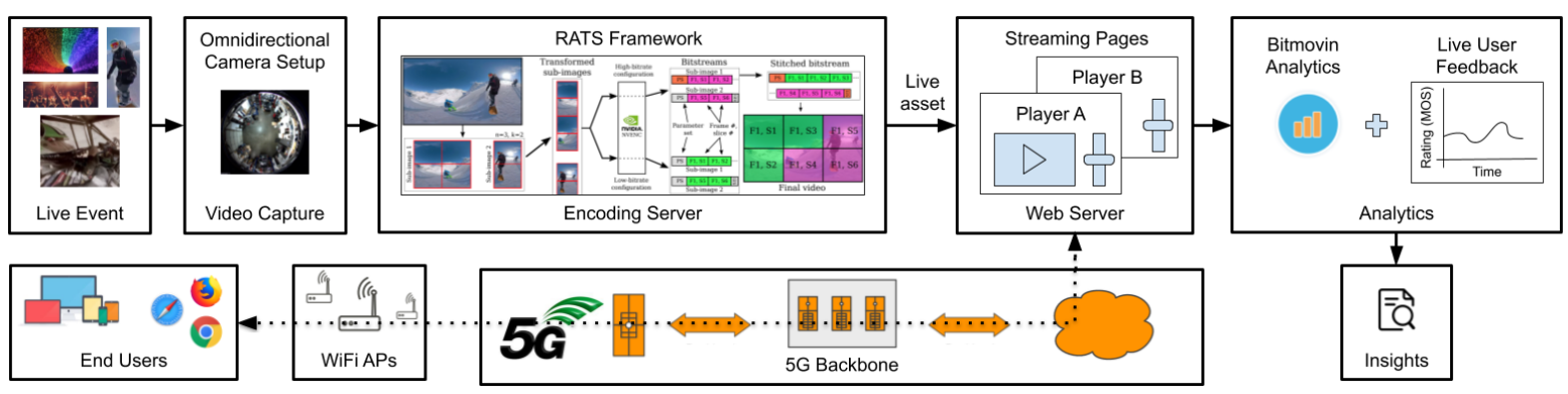

Figure 1: Proposed evaluation framework

\begin{abstract}
The end-to-end distribution of real-time $360^{\circ}$ video needs to be evaluated in a wholesome manner, considering all aspects from video capture to encoding, delivery, and playback, as well as timely and appropriate analytics, with a focus on enduser Quality of Experience (QoE). This requires a measurement framework which allows for the collection of metrics from multiple dimensions at the same time. In this work, we propose such a framework for evaluating real-time adaptive $360^{\circ}$ video streaming over an experimental Fifth Generation (5G) network, which allows for the investigation of different aspects of the end-to-end video delivery chain.
\end{abstract}

\section{KEYWORDS}

live streaming, mobile broadband, video analytics, QoE

\section{ACM Reference Format:}

Cise Midoglu, Ȯzgü Alay, and Carsten Griwodz. 2019. Evaluation Framework for Real-Time Adaptive 360-Degree Video Streaming over 5G Networks. In Wireless of the Students, by the Students, and for the Students 2019 Workshop (S3 '19), October 21, 2019, Los Cabos,

Permission to make digital or hard copies of part or all of this work for personal or classroom use is granted without fee provided that copies are not made or distributed for profit or commercial advantage and that copies bear this notice and the full citation on the first page. Copyrights for thirdparty components of this work must be honored. For all other uses, contact the owner/author(s).

S3 '19, October 21, 2019, Los Cabos, Mexico

(c) 2019 Copyright held by the owner/author(s).

ACM ISBN 978-1-4503-6929-9/19/10.

https://doi.org/10.1145/3349621.3355726
Mexico. ACM, New York, NY, USA, 3 pages. https://doi.org/10.1145/ 3349621.3355726

\section{INTRODUCTION}

360 -degree, or $360^{\circ}$, videos are recordings where a view in every direction is captured at the same time, shot using an omnidirectional camera or a collection of cameras. During playback, the viewer has control of the viewing direction through device controls or a specialized headset. $360^{\circ}$ video is growing in prominence, with big actors like Facebook ${ }^{1}$ and YouTube ${ }^{2}$ providing support since a number of years.

$360^{\circ}$ video streaming in Mobile Broadband (MBB) networks poses significant challenges in terms of bandwidth consumption and risk of stalling. Although recent approaches encompassing tiled adaptive streaming [2] alleviate some of these challenges for Video-on-Demand (VoD), most do not provide performance guarantees for real-time video delivery, where streaming live content exacerbates existing challenges.

With multiple points of potential failure/bottleneck in the delivery chain, the end-to-end distribution of real-time $360^{\circ}$ video needs to be evaluated in a wholesome manner, considering all aspects from video capture to encoding, delivery, and playback, as well as timely and appropriate analytics, with a focus on end-user Quality of Experience (QoE). This requires a measurement and experimentation framework which allows for the collection of metrics from multiple dimensions (such as encoding parameters, delivery network

\footnotetext{
${ }^{1}$ https://facebook360.fb.com

${ }^{2}$ https://support.google.com/youtube/answer/6178631
} 
information, player configuration and adaptation logic, enduser hardware/software, and objective/subjective QoE metrics for the video session) at the same time, as well as the benchmarking of selected parameters (such as different video tiling strategies, network technologies, video players, and bitrate adaptation algorithms). In this work, we propose such a framework for evaluating real-time adaptive $360^{\circ}$ video streaming over an experimental Fifth Generation (5G) network, which allows for investigating different aspects of the end-to-end video delivery chain. To the best of our knowledge, this is the first framework to combine real-time and $360^{\circ}$ streaming in next generation MBB networks with Overthe-Top (OTT) video analytics.

\section{FRAMEWORK}

Figure 1 presents our proposed framework. A live event (e.g., festival, concert, sports competition) is captured with an omnidirectional camera setup, and the feed is subsequently fed to the encoding server. The encoding server prepares the live stream for playback, and passes it to the web server. The web server incorporates multiple test pages with different video players, where viewers can provide continuous QoE feedback during their streaming session. Each page is integrated with an OTT video analytics module. End-users can stream the event over an experimental 5G network serving a designated test area. QoE feedback is collected from end-users in a crowdsourced manner, along with automatic video analytics data from the OTT module, and insights are derived regarding the performance of different encoding parameters, tiling strategies, video players, adaptation algorithms, and network technologies. Details regarding each framework component are provided below.

\subsection{Omnidirectional Video Capture}

Specialized hardware is required for the purpose of filming $360^{\circ}$ videos. We consider a high-end fisheye camera, which yields a rectangular compressed video at $12 M P$ with a black boundary around a circular recording. The camera delivers a single-lens image without other visual artifacts other than regular distortion. We capture the spherical video as a realtime byte-stream.

\subsection{Tiled Adaptive Bitrate Encoding}

Approaches to stream $360^{\circ}$ video adaptively (i.e., transferring only parts of the video such as the current and predicted viewport in high quality, while transferring the rest of the video tiles in lower quality) present significant bandwidth savings at little risk of stalling. Most recently, Ballard et al. [1] demonstrate a framework called Real-time Adaptive Three-sixty Streaming (RATS), where a GPU-based High Efficiency Video Coding (HEVC) encoding is utilized to tile, encode, and stitch $360^{\circ}$ video at different qualities in real time. They show measurement results for the encoding speed, amount of output data, and output quality for different tiling configurations, establishing that their approach is feasible for live streaming scenarios as well as VoD. In this work, we utilize the RATS framework and place it in a larger context of real-time adaptive $360^{\circ}$ video streaming over an experimental $5 \mathrm{G}$ network. The stitching is implemented on the server side, which allows for deployment in 5G edge computing scenarios.

\subsection{Live Streaming over 5G}

Multimedia experiments are often conducted in controlled lab environments, over fixed or emulated networks. However, these results do not always represent the mobile network conditions of "the wild". Although there are studies which have attempted to measure and model the performance characteristics of video streaming over operational MBB networks [5], these are limited to Fourth Generation (4G) networks. In this study, we target an experimental 5G deployment, giving us the opportunity to provide early feedback on the characteristics and performance of video delivery over $5 \mathrm{G}$ as compared to $4 \mathrm{G}$. We use the experimental $5 \mathrm{G}$ facility established as a part of the EU 5GENESIS project [4] in Berlin, Germany, which provides a $5 \mathrm{G}$ core with last-mile access over $\mathrm{WiFi}^{3}$. The WiFi Access Points (APs) are directly attached to computers that implement $5 \mathrm{G}$ edge nodes, which are connected to a $5 \mathrm{G}$ core network. Each of these APs create a dedicated network, where each end-user is assigned to a particular edge node. There are no handovers.

\subsection{Multiple Player Support}

$360^{\circ}$ videos can be viewed via mobile devices such as smartphones and tablets, where users can pan around the video by clicking and dragging. Internal sensors can also be used to pan the video based on the orientation of the device. However, the ultimate user experience depends on how well a video player exploits these features. There are numerous video players which claim to have $360^{\circ}$ support, however existing literature on the comparison of different players have mostly focused on VoD streaming [6]. In our framework, we provide test pages integrated with Video.js Player ${ }^{4}$ and Bitmovin Player ${ }^{5}$, in order to allow for the benchmarking of their real-time $360^{\circ}$ video streaming performance. The framework can easily be extended to support more players and player configurations.

\footnotetext{
${ }^{3}$ This platform aims at showcasing immersive service provisioning via advanced backhauling technologies, see https://5genesis.eu for details. ${ }^{4}$ https://videojs.com

${ }^{5}$ https://bitmovin.com/video-player/
} 


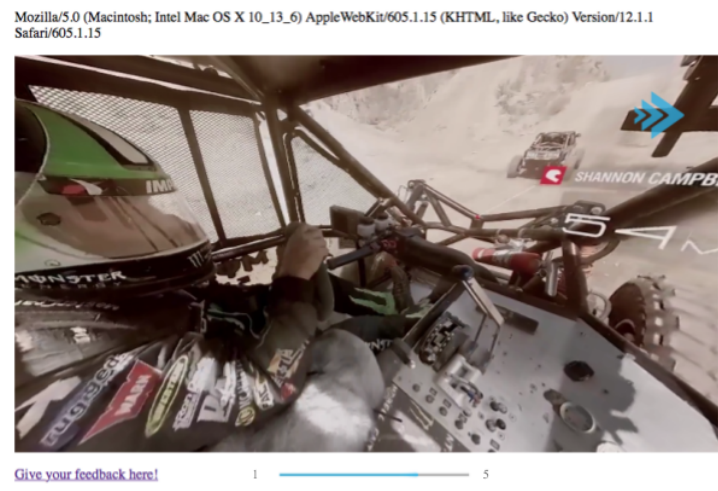

Figure 2: Test page integrating $360^{\circ}$ player with a slider bar on the bottom for continuous user feedback

\subsection{Crowdsourced QoE from End-Users}

On each of our test pages, we integrate interactive UI controls alongside the default viewing controls for $360^{\circ}$ video. The UI element is linked to a JavaScript code block that continuously collects timestamped user scores in Absolute Category Rating (ACR) format [3], and stores each time series with a distinct Universally Unique Identifier (UUID) at the end of the session, on the server side. This UUID can be matched to the session ID, which is used to differentiate between each session by the video analytics module. Figure 2 shows one of our test pages, including a sliding bar recording input continuously. The default is set to 3.5 on a $1-5$ scale, and the value is read every second.

\subsection{Video Analytics}

As part of our framework, we use a commercial OTT video analytics product to extract video performance metrics in real-time with low overhead. We select Bitmovin Analytics ${ }^{6}$, due to its easy integration with many open-source and commercial video players, large set of video performance metrics, user-friendly dashboard, and extensive developer documentation. The product is free to use up to $5000 \mathrm{im}$ pressions for any new user, and provides a cloud based data export service. Each session, for which there is analytics data, can be matched to the QoE scores provided by the end users using the Bitmovin Analytics session ID. Figure 3 presents a comparison page snippet from the Bitmovin dashboard.

\section{CONCLUSION}

We propose a measurement and evaluation framework for real-time adaptive $360^{\circ}$ video streaming over $5 \mathrm{G}$ mobile networks, which allows for the analysis of different influence factors on the end-to-end video delivery chain through crowdsourced QoE measurements. This framework can be used

\footnotetext{
${ }^{6}$ https://bitmovin.com/video-analytics/
}

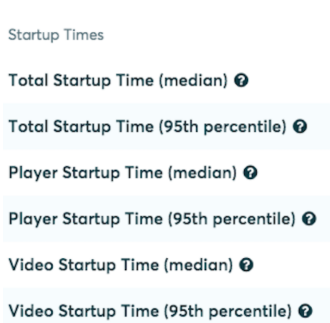

\begin{tabular}{|c|c|}
\hline bitmovin & videojs \\
\hline $0.81 \mathrm{~s}$ & $0.11 \mathrm{~s}$ \\
$3.31 \mathrm{~s}$ & $1.33 \mathrm{~s}$ \\
$0.38 \mathrm{~s}$ & $0.11 \mathrm{~s}$ \\
$1.77 \mathrm{~s}$ & $0.19 \mathrm{~s}$ \\
$0.25 \mathrm{~s}$ & $0.02 \mathrm{~s}$ \\
$2.44 \mathrm{~s}$ & $2.12 \mathrm{~s}$ \\
\hline
\end{tabular}

Figure 3: Sample video player comparison, available from the OTT video analytics module dashboard

by researchers and other stakeholders to benchmark video streaming performance in different networks, with different encoding and tiling parameters, video players, bitrate adaptation algorithms, network configurations, and end-user devices (in terms of hardware, e.g., tiling support, and in terms of software, e.g, compatibility of operating system and browser model/version with $360^{\circ}$ playback). Insights about QoE can also be derived by combining objective session metrics with subjective end-user scores.

A live demonstration of the framework is possible, where the client side can be emulated by any end-user device with broadband Internet access. It is possible to provide QoE feedback using any browser that supports JavaScript MSE and HEVC decoding (a non-tiled video stream encoded with H.264 is also available for other browsers). The server side is prepared with a built-in consumer grade GPU and is available remotely. Video quality metrics and benchmarks are available from the analytics integration in real-time.

\section{ACKNOWLEDGMENTS}

This work was funded by the EU H2020 Research and Innovation Programme under grant agreement No. 815178, and the German Research Foundation (DFG) as part of SFB 1053 "MAKI".

\section{REFERENCES}

[1] T. Ballard et al. RATS: Adaptive 360-degree Live Streaming. International Conference on Multimedia Systems (MMSys), 2019.

[2] M. Graf et al. Towards Bandwidth Efficient Adaptive Streaming of Omnidirectional Video over HTTP: Design, Implementation, and Evaluation. International Conference on Multimedia Systems (MMSys), 2017.

[3] International Telecommunication Union. Methods for subjective determination of transmission quality. Recommendation P.800, 1996.

[4] H. Koumaras et al. 5GENESIS: The Genesis of a flexible 5G Facility. International Workshop on Computer-Aided Modeling Analysis and Design of Communication Links and Networks (CAMAD), 2018.

[5] A. Schwind et al. Dissecting the performance of YouTube video streaming in mobile networks. International fournal of Network Management (IfNM), 2019.

[6] A. Zabrovskiy et al. AdViSE: Adaptive Video Streaming Evaluation Framework for the Automated Testing of Media Players. International Conference on Multimedia Systems (MMSys), 2017. 